\title{
Hospice Care in Long-Term Care Facilities
}

\author{
Ya-Lie Ku* \\ Department of Nursing, Taiwan
}

ISSN: 2578-0093

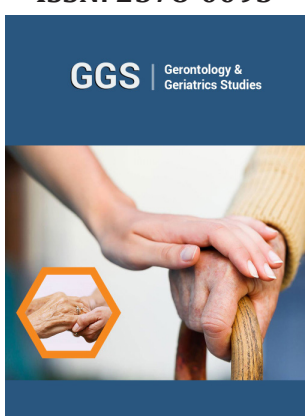

*Corresponding author: Ya-Lie $\mathrm{Ku}$, Department of Nursing, Taiwan

Submission: 韭 February 18, 2019

Published: 眥March 13, 2019

Volume 4 - Issue 4

How to cite this article: Ya-Lie K. Hospice Care in Long-Term Care Facilities. Gerontol \& Geriatric stud.4(4). GGS.000591.2019. DOI: 10.31031/GGS.2019.04.000591

Copyright@ Ya-Lie K, This article is distributed under the terms of the Creative Commons Attribution 4.0 International License, which permits unrestricted use and redistribution provided that the original author and source are credited.

\section{Introduction}

Aging societies are a global phenomenon, one which Taiwan is experiencing. Taiwan has been an aging society since 1993; it became an aged society in 2018 and is predicted to be a super-aged society by 2026 [1,2]. Because of the busy lives of their children, aging or disabled elders in Taiwan often live in long-term care facilities, without support systems. Such elders do not know when or how they will die. Moreover, they are unequipped to handle the dying process of their family members and other elders in such facilities. Long-term care nurses and elders are frequently stoic about the death of elders living with them the facilities. Dying and death are taboo and secret topics for long-term care facilities, since the elders died is the normal process in one's life toward both the workers and survived elders. However, when a 60 -year-old person encounters the death of a 90 -year-old person, they may fear that they are next. How do the surviving elders experience such an ambiguous and fearful phenomenon?

Not all countries have do not resuscitate (DNR) or advanced directive (AD), which can be approved in advance by elderly people in long-term care facilities; in such cases, the elderly people and their family members need not be concerned about decisions surrounding elderly people who are facing death. For example, in six European countries, end-of-life decisionmaking frequently precede dying, and patients and relatives are generally involved in this decision-making process [3]. The AD plan can foster knowledge and healthy approaches among elderly people in Taiwan, among other countries, thus allowing them autonomy over the decision-making process, and nurses can actively promote the hospice care and AD plans for elderly people [4] and motivate elderly people with renal dialysis to approve the AD sooner than later [5]. In addition, if family members and the healthcare team arrange the endof-life plan relatively early for elderly patients, the psychological stress and medical expenses for those involved might be reduced [6]. For example, in the case of an elderly patient with terminal dementia who was dependent on the machine for a long period of time, the family members had approved the DNR in case of respiratory failure, ensuring that the patient can die without unnecessary suffering and burden on family members [7].

The process of life and death goes beyond merely signing a DNR or AD for elderly people. More can be done for elderly people and their family as well as for those who work in longterm care facilities. The people involved do not have to face the process of death reticently. In all societies, children are provided life education. Can we provide such life education for the surviving elders, healthy or otherwise, by teaching them to have an optimistic perspective and providing approaches to handle their death? Do we have role models of long-term care facilities? Can we merge alternative activities, such as intimacy touch, music, art, horticultural, reminiscence, cognitive therapies, and psychotherapy, with life education for elderly people to spread awareness and help them cope with death? For example, Taoist and Confucian philosophies strongly influence the perspectives of elderly people on life in Taiwan $[8,9]$. Can we merge Chinese literature or philosophy with the hospice care for elderly people?

For example, nurses could help elderly patients with liver cirrhosis to conduct their life review by recalling their meaningful life moments and assist them in accepting and valuing themselves to achieve life integrity [10]. Nevertheless, considering the behavior of the public and healthcare professionals, finding effective approaches of encouraging dialogue and choice within the constraints of the current healthcare systems and personal 
circumstances is difficult for AD discussions with frail and elderly people [11]. Moreover, studies have shown that nursing home hospice collaborations require effective communication regarding changing care requirements, and a range of barriers can impede the integration of hospice and nursing home care [12]. Therefore, the [13], developed the Catholic Sanipax Socio-Medical Service \& Education Foundation in Taiwan for healthcare professionals and family members, can be followed when providing hospice care for terminal patients and elderly people. The brochure includes an unfinished will, monetary arrangements, a photograph, ceremonies, rituals, burial methods, and messages for others, all of which could be completed by terminal patients or elders in advance so that family members and friends could help and assist them to complete their wishes.

The provision of nursing home hospice has positive effects on non-hospice residents, which provides indirect benefits to nursing home clinical practitioners Stevenson [12]. Qualitative thematic evidence has revealed that hospice daycare services generate a renewed meaning and purpose for not only the nursing home but also terminal patients, and home hospice services support families to sustain patient care at home [14]. The relevant literature indicates that a conversation about death with terminal patients and family members is the primary concern for long-term care providers. A protocol for healthcare providers to converse about death with elderly people through alternative and innovative activities must be made available in the long-term care facilities. In the long term, elderly people and caretakers in long-term care facilities should spontaneously and peacefully cope with the death of other elderly people and themselves through verbal and nonverbal behaviors..

\section{References}

1. Department of Statistics (2016) Monthly report of the interior statistics.

2. National Development Council (2016) Population estimate of Taiwan (2016- 2061).
3. van der Heide A, Deliens L, Faisst K, Nilstun T, Norup M, et al. (2003) End-of-life decision-making in six European countries: descriptive study. Lancet 362(9381): 345-350.

4. Lin CY, Sung HC, Fan SY, Wang SC, Hu HF (2014) The effects of the advance care planning program on knowledge and attitude of hospice care and advanced directives among older adults in a long-term care facility: A pilot study. Chung Gang Nursing 25(4): 389-403.

5. Chen SY, Hu WY, Yu Y, Kao TW, Ho CI (2011) Palliative care in elderly with end-stage renal disease under dialysis. Formosa Journal of Medicine 15: 311-319.

6. Chang L, Huang SJ, Hsiao SH (2015) From ICU return to end-of-life care plan. Taipei City Medicine 12(1): 13-27.

7. Chiu LF, Tseng HL, Li JP, Wang NT, Hung TC (2011) Adopting hospice care to help the ventilator-dependent patients pass their end-stage course. Taiwan Journal of Hospice Palliative Care 16(2): 228-239.

8. Lee HT, Chen CY, Hu WY (2012) Advance care planning and elderly autonomy among long-term care facilities residents under chinese filial piety and familism culture. Taiwan Journal of Hospice Palliative Care 17(2): 187-199.

9. Hsin DH, Macer D (2006) Comparisons of life images and end-of-life attitudes between the elderly in Taiwan and New Zealand. Journal of Nursing Research 14(3): 198-208.

10. Wu HY, Huang HC (2013) The care experience of facing to death in a community of the elderly with end-stage liver cirrhosis through "life review". Journal of Mackay Nursing 8: 85-94.

11. Sharp T, Moran E, Kuhn I, Barclay S (2013) Do the elderly have a voice? Advance care planning discussions with frail and older individuals: A systematic literature review and narrative synthesis. British Journal of General Practice 63(615): e657-e668.

12. Stevenson DG, Bramson JS (2009) Hospice care in the nursing home setting: A review of the literature. Journal of Pain and Symptom Management 38(3): 440-451.

13. Life Album (2015) Catholic sanipax socio-medical service \& education foundation.

14. Candy B, Holman A, Leurent B, Davis S, Jones L (2011) Hospice care delivered at home, in nursing homes and in dedicated hospice facilities: A systematic review of quantitative and qualitative evidence. Internal Journal of Nursing Studies 48(1): 121-133. 